Casper W. Bollen

Cuno S. P. M. Uiterwaal

Adrianus J. van Vught

\section{Meta-regression analysis of high-frequency ventilation vs conventional ventilation in infant respiratory distress syndrome}

Received: 10 January 2007

Accepted: 11 January 2007

Published online: 24 February 2007

(C) Springer-Verlag 2007

Electronic supplementary material The online version of this article (doi:10.1007/s00134-007-0545-y) contains supplementary material, which is available to authorized users
C. W. Bollen ( $)$ A A. J. van Vught University Medical Center Utrecht, Pediatric Intensive Care Unit, 85090, 3508 AB Utrecht, The Netherlands e-mail: c.w.bollen@umcutrecht.nl Tel.: +31-30-2504002

Fax: +31-30-2505340

C.S.P. M. Uiterwaal

University Medical Center Utrecht, Julius Center for Health Sciences and Primary Care,

85500, 3508 GA Utrecht, The Netherlands

\begin{abstract}
Objective: There is considerable heterogeneity among randomized trials comparing highfrequency ventilation (HFV) with conventional mechanical ventilation (CMV) in premature neonates with respiratory distress syndrome. We investigated what factors explained differences in outcome among these trials. Design: Meta-regression analysis of 15 randomized trials. Measurements and results: Variables were extracted to explain heterogeneity: year of publication; use of Sensormedics 3100A ventilator for $\mathrm{HFV}$; time on CMV prior to start of study; gestational age; use of surfactant; high lung volume strategy in HFV; and lung protective ventilation strategy in CMV and baseline risk. Chronic lung disease (CLD) and death or CLD were outcome measures. Relative risk ratios were calculated to estimate effect sizes of explanatory variables on reported relative risks. Adjusted estimates of relative risk ratios of high lung
\end{abstract}

volume strategy and lung protective ventilation strategy were $0.42(95 \%$ CI $0.06-2.48)$ and 2.02 (95\% CI $0.18-23.12$ ) for CLD, respectively. The effect of gestational age was less pronounced $(\mathrm{RRR}=1.17(95 \% \mathrm{CI}$ 0.16-8.32) for CLD, respectively). Use of Sensormedics and prior time on CMV had the smallest effects $[R R R=0.96(95 \%$ CI $0.47-1.94)$ and $\mathrm{RRR}=0.85$ (95\% CI 0.58-1.24) for CLD, respectively)]. The same results applied to CLD or death as outcome. Conclusions: Variation in ventilation strategies that were used in trials comparing HFV with CMV in premature neonates offered the most likely explanation for the observed differences in the outcome of these trials compared with other explanatory factors.

Keywords Conventional mechanical ventilation - High-frequency ventilation - Meta-regression analysis - Respiratory distress syndrome $\cdot$ Infant

\section{Introduction}

High-frequency ventilation (HFV) has been compared with conventional mechanical ventilation (CMV) since the 1980s. In HFV, patients are ventilated with small tidal volumes, even smaller than the dead space of their airways, at high frequencies, normally between 5 and $10 \mathrm{~Hz}$. Because HFV combines high mean airway pressures with small tidal volumes, this technique of ventilation has been regarded by some to be the most optimal form in patients with infant respiratory distress syndrome (IRDS), adult respiratory distress syndrome (ARDS), and other forms of severe lung disease [1].

The HFV has been extensively investigated in premature neonates with IRDS, a population specifically at risk for chronic lung disease (CLD). Unfortunately, the results of these studies were equivocal $[2,3]$; thus, the question remains whether or not HFV better prevents CLD than conventional mechanical ventilation (CMV) in patients with severe lung disease. A significant number of meta- 
analyses have been performed to answer this question $[4$, $5,6,7,8]$. Pooled estimates of pulmonary outcomes failed to show clinically relevant differences among HFV and CMV [7]; however, significant heterogeneity existed between studies included in these meta-analyses. In a recent cumulative meta-analysis we identified improvements of the conventional treatment of IRDS and ventilation strategies applied in both HFV and CMV as important sources of heterogeneity [4]. These associations could be confounded by other explanatory variables. Although a meta-analysis may pool results from randomized trials, differences among trials will not be randomly or independently distributed. A meta-analysis constitutes an observational study of trials, subjected to bias inherent to observational research. In a meta-regression analysis it is possible to adjust for confounding covariates. A number of alternative hypotheses have been formulated to explain heterogeneity between trials:

1. The observed regression of the cumulative relative risks to the level of unity was due to publication bias.

2. Use of the Sensormedics ventilator resulted in better results in HFV treated patients.

3. A prolonged ventilation on CMV before initiating HFV treatment could reduce the benefits of HFV.

4. In subgroups of more premature neonates with lower birth weight with a higher susceptibility for CLD, HFV could result in better pulmonary outcome.

5 . With outcome rates increasingly representing more severe disease, HFV could have an increasing advantage over CMV [9, 10]; therefore, we used meta-regression analysis to better estimate relative treatment effects through adjustments for factors that could explain trial heterogeneity.

\section{Methods}

Trials were included based on a previous meta-analysis that we had conducted [4]. The same search strategy, as well as the same inclusion and exclusion criteria as in our previous meta-analysis, were used for an update, yielding two more studies that could be included for this metaregression analysis. Validity of studies was assessed by criteria published by Jadad et al. [11]. The validity was generally deemed as high with adequate allocation concealment in all trials. Blinding of treatment was not possible due to the nature of the interventions.

Data extraction was performed as has been reported in our previous meta-analysis. The following outcome measures were used: mortality, chronic lung disease (CLD) as defined by supplemental oxygen need or ventilator dependency at the age of 30-36 weeks post-menstrual. A number of explanatory variables were extracted as well: year of publication; type of ventilator used for HFV (Sensormedics 3100A ventilator versus other); ventilation strategies applied in the HFV and CMV treatment groups were obtained as previously described [4]; time on CMV before study initiation; gestational age and birth weight; and outcome rates in the control population were taken as proxy for baseline disease severity in the source population. The Sensormedics ventilator was singled out because previous research suggested better performance compared with other oscillator ventilators $[2,4]$.

\section{Statistical analysis}

All data were extracted according to the intention-to-treat principle. The number of patients surviving without chronic lung disease was subtracted from the total number of randomized patients in each treatment arm to calculate the composite outcome of death or CLD. To calculate the risk of CLD, the number of surviving patients was put in the denominator. Publication bias was assessed by visual appraisal of symmetry of funnel plots and performing rank tests. Smaller studies could show different results than larger studies which could suggest publication bias, but in fact was caused by systematic differences among studies; therefore, an analysis of publication bias stratified for ventilation strategies was performed to determine whether the observed association between the inverse of the standard error with the risk ratio was confounded by ventilation strategies. Meta-regression analysis was used to evaluate other hypotheses. The dependent variables, RR of CLD and RR of CLD or death, were natural log transformed to linearize the regression models. Individual studies were weighted by inverse variances of relative risks of outcomes of interest so that the more precise studies had more influence in the analysis. Firstly, linear regression analyses were applied to explanatory variables. Secondly, linear regression analyses with continuous covariates were conducted stratified by HLVS, LPVS, and use of surfactant. Finally, multivariable linear regression analyses were performed to calculate adjusted contributions of different explanatory variables of rivalling hypotheses to changes in RR. The relative effects of covariates were evaluated by relative risk ratios (RRR). A relative risk ratio quantifies the relative change in RR that is associated with a specified change of a covariate. For continuous variables the RRR was calculated for the ranges of minimum and maximum values of covariates that were reported in trials. For example, the RRR for year of publication was calculated by using the range between the publication year of the first year and the publication year of the last included trial. The RRR for year of publication thus estimates the relative change in RR due to the difference in years of publication between the first and last trials. All analyses were conducted using 
SPSS 12.0.1 for Windows software (SPSS, Chicago, Ill.) and Excel (Microsoft, Redmond, Wash.).

\section{Results}

For the analyses 15 studies were available that specified either CLD in survivors or death or CLD as outcome measures $[2,3,12,13,14,15,16,17,18,19,20,21,22,23$, 24]. In 11 trials a high frequency oscillatory ventilator was used $[2,3,12,13,16,17,19,20,22,23,24]$, in 7 of these trials this was the SensorMedics ventilator $[2,12,13$, $17,22,23]$. Two studies used a high-frequency jet ventilator $[14,15]$ and in two studies a high-frequency flow interrupter ventilator was used $[18,21]$. In the HFV group a total of 1141 patients were included for the outcome of CLD with 373 events and a total of 1457 patients with 671 events for the outcome death or CLD. In the CMV group a total 1159 patients were reported for the outcome of CLD with 428 events and a total of 1473 patients with 730 events for the outcome death or CLD. A forest plot of these trials can be found in the Electronic Supplement.

Table 1 summarizes the main characteristics of the studies. The outcome of CLD was available in all studies but one [22]. Time on CMV to start of the study was not reported by Plavka et al. [17] and Craft et al. [21]. In only one study was surfactant not used as concomitant treatment [12]. A high lung volume strategy (HLVS) was used in all but two studies $[14,16]$. A ventilation strategy in the CMV-treated patients that could qualify as lung protective (LPVS) was reported in the most recent 9 studies [2, 3, 18, 19, 20, 21, 22, 23, 24]. Studies were published over a range of 13 years. Other reported ranges of covariates were $8.7 \mathrm{~h}$ average time on CMV before start of study, 5 weeks average gestational age, and $0.65 \mathrm{~kg}$ average birth weight. These ranges were used to calculate RRRs. Two studies dominated the analyses by virtue of the weight they received in the analyses: Johnson et al. [3] and Courtney et al. [2] (together 69\% for CLD and 73\% for death or CLD as outcome).

A funnel plot of the inverse of the standard error against the natural logarithm of the RR for CLD was indicative of publication bias because of asymmetry round the line of the pooled effect (Fig. 1). A rank test showed a $p$-value of 0.112 . A stratified analysis of publication bias is indicated by different colors in Fig. 1. To visually evaluate publication bias within subgroups of ventilation strategy, the distribution of trials round the corresponding colored lines (mean effect size within subgroup) was assessed. Stratification by ventilation strategy (HLVS and LPVS vs either no HLVS and/or no LPVS) showed $p$-values of 0.456 and 0.851 , respectively, indicating less evidence of publication bias. The distribution of stratified studies round the lines of pooled estimates showed less asymmetry (Fig. 1). Publication bias for the composite outcome of death or CLD was less likely with a $p$-value of 0.329 . Stratified analysis showed $p$-values of 0.677 and 1.000 .

Figures 2, 3, 4 show the results of the linear metaregression analyses for continuous explanatory variables with relative risk of CLD as dependent variable. Two studies dominate these figures, designated by the weight they received in the analyses [2,3]. Over the years the reported benefit of HFV over CMV seemed to diminish (Fig. 2). A longer time on CMV prior to study initiation

Table 1 Study characteristics

\begin{tabular}{|c|c|c|c|c|c|c|c|c|c|c|c|c|}
\hline Reference & Year & $\begin{array}{l}\text { Time on } \\
\text { CMV }\end{array}$ & Age & $\begin{array}{l}\text { Birth } \\
\text { weight }\end{array}$ & SensorM & HLVS & LPVS & Surf & $\begin{array}{l}\text { CLD } \\
\operatorname{lnRR}\end{array}$ & Weight & $\begin{array}{l}\text { Death } \\
\text { or CLD } \\
\text { lnRR }\end{array}$ & Weight \\
\hline [12] & 1992 & 9.0 & 28 & 1.100 & Y & Y & $\mathrm{N}$ & $\mathrm{N}$ & -1.29 & 0.01 & -0.58 & 0.01 \\
\hline [13] & 1996 & 3.0 & 31 & 1.500 & Y & Y & $\mathrm{N}$ & Y & -0.67 & 0.04 & -0.55 & 0.02 \\
\hline [14] & 1996 & 7.2 & 27 & 0.950 & $\mathrm{~N}$ & $\mathrm{~N}$ & $\mathrm{~N}$ & $\mathrm{Y}$ & 0.02 & 0.01 & -0.23 & 0.10 \\
\hline [15] & 1997 & 8.0 & 27 & 1.020 & $\mathrm{~N}$ & Y & $\mathrm{N}$ & Y & -0.70 & 0.03 & 0.48 & 0.03 \\
\hline [16] & 1998 & 1.0 & 28 & 1.100 & $\mathrm{~N}$ & $\mathrm{~N}$ & $\mathrm{~N}$ & Y & 0.00 & 0.00 & 0.31 & 0.00 \\
\hline [17] & 1999 & & 26 & 0.850 & Y & Y & $\mathrm{N}$ & Y & -1.03 & 0.01 & -0.74 & 0.01 \\
\hline [18] & 1999 & 0.5 & 27 & 0.870 & $\mathrm{~N}$ & Y & Y & Y & 0.09 & 0.06 & 0.01 & 0.04 \\
\hline [19] & 2001 & 2.6 & 26 & 0.840 & Y & $\mathrm{Y}$ & Y & Y & -0.98 & 0.02 & -0.59 & 0.02 \\
\hline [20] & 2001 & 0.3 & 28 & 0.990 & $\mathrm{~N}$ & Y & Y & Y & -0.20 & 0.05 & -0.06 & 0.05 \\
\hline [2] & 2002 & 2.7 & 26 & 0.850 & Y & Y & Y & Y & -0.06 & 0.16 & -0.22 & 0.13 \\
\hline [3] & 2002 & 1.0 & 26 & 0.850 & $\mathrm{~N}$ & Y & Y & Y & -0.01 & 0.54 & -0.02 & 0.60 \\
\hline [23] & 2003 & 1.0 & 29 & 1.200 & Y & Y & Y & Y & 0.32 & 0.03 & 0.27 & 0.04 \\
\hline [22] & 2003 & 14.0 & 27 & 0.980 & Y & $\mathrm{Y}$ & Y & Y & & & -0.04 & 0.05 \\
\hline [21] & 2003 & & 26 & 0.726 & $\mathrm{~N}$ & Y & Y & Y & 0.10 & 0.05 & 0.09 & 0.03 \\
\hline [24] & 2005 & 0.3 & 27 & 0.880 & $\mathrm{~N}$ & $\mathrm{Y}$ & Y & Y & -1.44 & 0.01 & -1.20 & 0.00 \\
\hline
\end{tabular}

Year: year of publication; Time CMV: Mean time on CMV before start of the study in hours; Age: Mean gestational age (weeks); Birth weight: mean birth weight $(\mathrm{kg}) ; H L V S$ : high lung volume strategy in the HFV group; LPVS: lung protective ventilation strategy in the CMV group; Surf: use of surfactant in the study; $C L D$ : chronic lung disease, defined as on oxygen at 30-36 weeks postmenstrual age; LnRR: natural $\log$ of the relative risk 


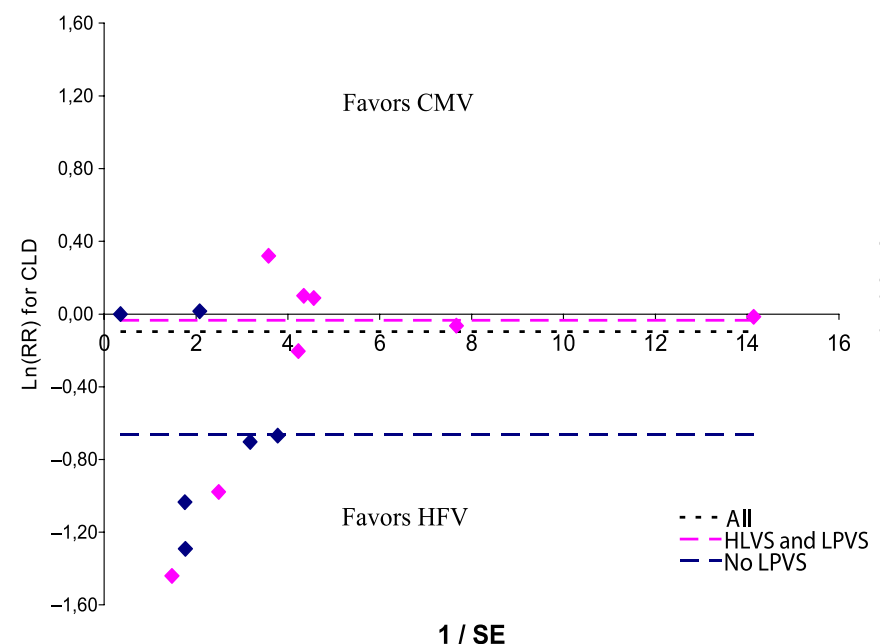

Fig. 1 Funnel plot. Selection bias in reporting RR of chronic lung disease (CLD) as suggested by asymmetry of the distribution of studies. $x$-axis: inverse of the standard error of the RR; $y$-axis: natural logarithm of the RR. Blue diamonds: studies with either no high lung volume strategy $(H L V S)$ or no lung protective volume strategy (LPVS); pink diamonds: studies with both HLVS and LPVS; dotted line: estimated pooled RR including all studies; dashed colored lines: pooled RR of subgroups of studies. Publication bias was visually appraised by assessing symmetry of distribution of studies around the lines of pooled estimates. CMV conventional mechanical ventilation

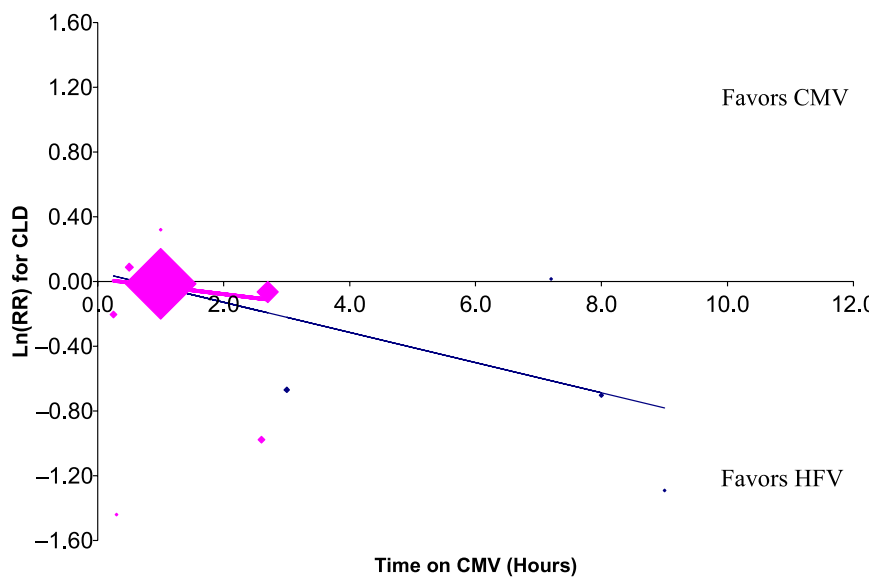

Fig. 3 Same as Fig. 2

and a higher gestational age and increase of birth weight (data not shown) seemed to be positively associated with a relatively better outcome in HFV (Figs. 3, 4). Table 2 shows the results of linear meta-regression analyses, showing significant associations with year of publication (3.1 times higher RR with change of publication year from 1992 to 2005) and whether or not a protective ventilation strategy was applied (1.9 times higher RR with change of protective ventilation from no to yes; Table 2). In the linear regression analyses with death or CLD as composite outcome no significant associations were detected. Whether or not a Sensormedics high-frequency

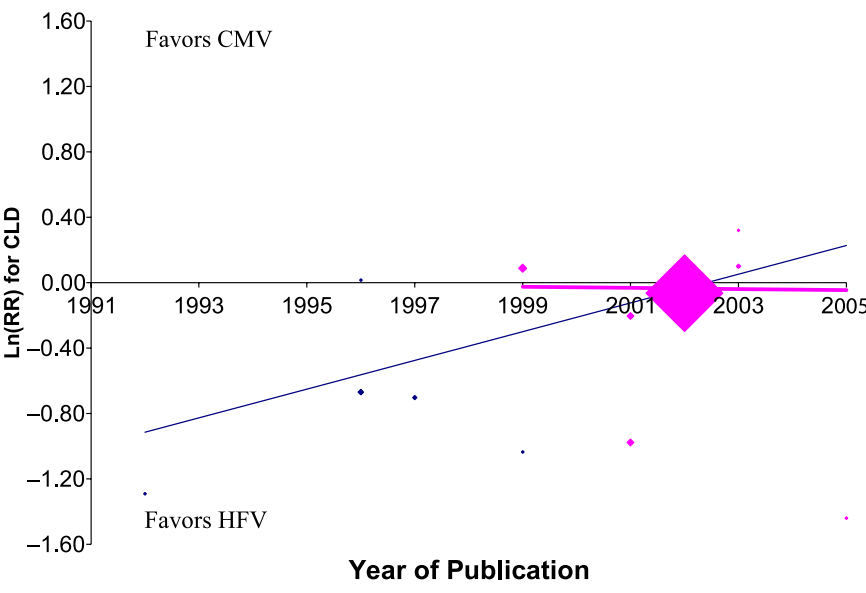

Fig. 2 Linear regression analyses. Crude and subgroup linear regression analyses of the effect of year of publication, prior time on CMV and gestational age with natural logarithm of RR of CLD as dependent variable. $y$-axis: natural logarithm of the RR; $x$-axis: explanatory variables. Blue diamonds: studies with either no HLVS or no LPVS; pink diamonds: studies with both HLVS and LPVS. The size of the diamonds reflects the weights the individual trials contribute to the analyses. Thin blue line: regression line including all studies; thick pink line: regression line including only studies with both HLVS and LPVS. $C M V$ conventional mechanical ventilation

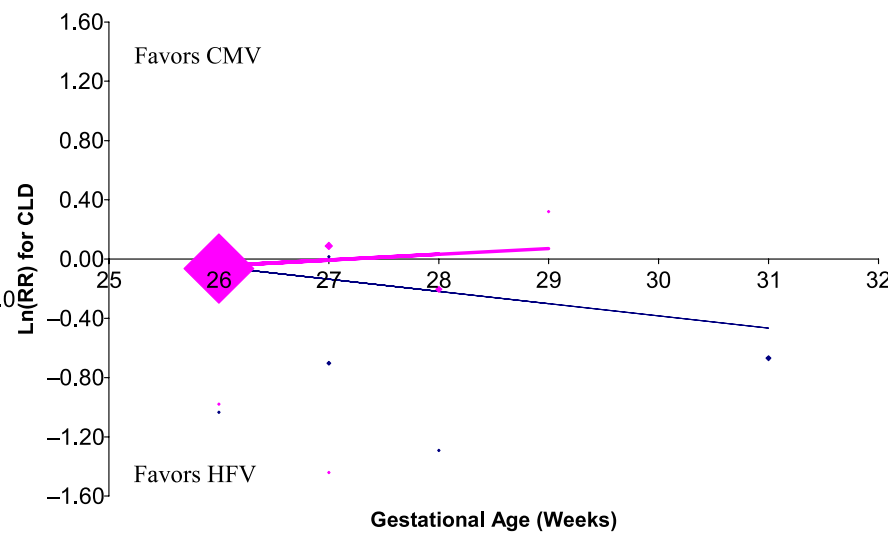

Fig. 4 Same as Fig. 2

oscillatory ventilator was used and baseline incidence in CMV (0.75 vs 0.08) treated patients displayed the smallest effects on trial outcome $(\mathrm{RRR}=0.84$ and 0.90 for CLD and $R R R=0.85$ and 0.99 for death or CLD, respectively).

Figure 5 shows how the incidence of CLD in the CMV treated patients was related to the incidence in HFV treated patients for each of the studies. The diagonal line represents the line of no effect in this figure. A trend line was fitted by weighted linear regression, showing a small effect of change in incidence in CMV on incidence in HFVtreated patients. 
Year of publication was not related to change in relative risk of CLD in the subgroup of studies with HLVS, LPVS and concomitant use of surfactant $(\mathrm{RRR}=0.96)$. There was only a small increase in relative risk for death or CLD (RRR = 1.20; Fig. 2; Table 2). Opposite effects of gestational age $(\mathrm{RRR}=1.22$ for CLD and 1.38 for death or CLD vs $R R R=0.66$ for CLD and 0.91 for death or CLD, respectively) and birth weight were detected in the subgroup analysis (Fig. 4; Table 2). Prior time on CMV exerted less effect on outcome compared with the crude analysis, $\mathrm{RRR}=0.66$ for CLD and 0.96 for death or CLD and RRR $=0.44$ for CLD and 0.92 for death or CLD in the adjusted and crude analyses, respectively (Fig. 3; Table 2).

Multivariable linear regression analyses were conducted to assess the independent contributions to change in RR by explanatory variables (Table 3 ). The RRRs in Table 3 have the same meaning as in Table 2, only they represented adjusted RRRs. Year of publication was not considered as an independent explanatory variable but rather as proxy for changes in treatment and patient population. Gestational age and birth weight were collinearly related by nature; only gestational age

Table 2 Univariable linear regression analysis

\begin{tabular}{|c|c|c|c|c|c|c|c|}
\hline & \multirow[b]{2}{*}{ Crude B } & \multirow[b]{2}{*}{ Sig. } & \multicolumn{2}{|c|}{$95 \%$ confidence interval } & \multirow[b]{2}{*}{ RRR } & \multicolumn{2}{|c|}{$95 \%$ confidence interval } \\
\hline & & & $\begin{array}{l}\text { Lower } \\
\text { boundary }\end{array}$ & $\begin{array}{l}\text { Upper } \\
\text { boundary }\end{array}$ & & $\begin{array}{l}\text { Lower } \\
\text { boundary }\end{array}$ & $\begin{array}{l}\text { Upper } \\
\text { boundary }\end{array}$ \\
\hline & \multicolumn{7}{|c|}{ All studies } \\
\hline \multicolumn{8}{|l|}{$C L D$} \\
\hline Year & 0.09 & 0.025 & 0.01 & 0.16 & 3.13 & 1.18 & 8.27 \\
\hline $\begin{array}{l}\text { SensorM } \\
\text { (no to yes) }\end{array}$ & -0.17 & 0.351 & -0.55 & 0.21 & 0.84 & 0.58 & 1.24 \\
\hline TimeCMV & -0.09 & 0.055 & -0.19 & 0.00 & 0.44 & 0.19 & 1.02 \\
\hline Age & -0.08 & 0.237 & -0.23 & 0.06 & 0.66 & 0.32 & 1.36 \\
\hline Weight & -0.76 & 0.163 & -1.87 & 0.35 & 0.54 & 0.22 & 1.33 \\
\hline HLVS & -0.11 & 0.883 & -1.74 & 1.52 & 0.89 & 0.17 & 4.57 \\
\hline LPVS & 0.64 & 0.009 & 0.19 & 1.10 & 1.91 & 1.21 & 3.00 \\
\hline Surf & 1.21 & 0.168 & -0.59 & 3.00 & 3.34 & 0.56 & 20.03 \\
\hline CMV & -0.18 & 0.774 & -1.53 & 1.17 & 0.90 & 0.42 & 1.92 \\
\hline \multicolumn{8}{|l|}{ Death or CLD } \\
\hline Year & 0.05 & 0.096 & -0.01 & 0.12 & 2.01 & 0.86 & 4.65 \\
\hline SensorM & -0.17 & 0.132 & -0.39 & 0.06 & 0.85 & 0.67 & 1.06 \\
\hline TimeCMV & -0.01 & 0.590 & -0.05 & 0.03 & 0.92 & 0.65 & 1.29 \\
\hline Age & -0.02 & 0.733 & -0.13 & 0.10 & 0.91 & 0.52 & 1.61 \\
\hline Weight & -0.22 & 0.611 & -1.16 & 0.71 & 0.84 & 0.40 & 1.77 \\
\hline HLVS & -0.37 & 0.698 & -2.44 & 1.69 & 0.69 & 0.09 & 5.45 \\
\hline LPVS & 0.19 & 0.275 & -0.18 & 0.56 & 1.21 & 0.84 & 1.76 \\
\hline Surf & 0.52 & 0.289 & -0.51 & 1.56 & 1.69 & 0.60 & 4.75 \\
\hline CMV & -0.02 & 0.963 & -0.91 & 0.87 & 0.99 & 0.60 & 1.63 \\
\hline & \multicolumn{7}{|c|}{ Studies with surfactant, HLVS, and LPVS } \\
\hline$C L D$ & & & & & & & \\
\hline Year & 0.00 & 0.971 & -0.23 & 0.22 & 0.96 & 0.05 & 17.34 \\
\hline TimeCMV & -0.05 & 0.698 & -0.34 & 0.25 & 0.66 & 0.05 & 8.75 \\
\hline Age & 0.04 & 0.727 & -0.22 & 0.30 & 1.22 & 0.33 & 4.49 \\
\hline Weight & 0.41 & 0.693 & -1.99 & 2.81 & 1.38 & 0.20 & 9.44 \\
\hline \multicolumn{8}{|l|}{ Death or CLD } \\
\hline Year & 0.01 & 0.846 & -0.15 & 0.17 & 1.20 & 0.15 & 9.72 \\
\hline TimeCMV & 0.00 & 0.819 & -0.05 & 0.04 & 0.96 & 0.65 & 1.43 \\
\hline Age & 0.06 & 0.406 & -0.10 & 0.21 & 1.34 & 0.61 & 2.92 \\
\hline Weight & 0.55 & 0.396 & -0.89 & 1.99 & 1.55 & 0.49 & 4.90 \\
\hline
\end{tabular}

Simple linear regression analyses were calculated for chronic lung disease (CLD), defined as on oxygen at 30-36 weeks postgestational age, and death or CLD. The following co-variates were evaluated: Year: Number of years after the first included study; SensorM: Whether or not a Sensormedics type of HFV was used; TimeCMV: Mean time on CMV before start of the study in hours; Age: Mean gestational age (weeks); Weight: mean birth weight $(\mathrm{kg}) ; H L V S$ : high lung volume strategy in the HFV group; LPVS: lung protective ventilation strategy in the CMV group; Surf: use of surfactant in the study; B was the estimated crude coefficient; RRR: relative risk ratio $=\mathrm{RR}_{\text {covariate }=1} / \mathrm{RR}_{\text {covariate }=0}$, for binary variables (SensorM, HLVS and LPVS), for continues variables the extreme values reported in the studies were used to calculate the ranges, 13 for years, 8.7 for Time on $C M V$, 5 for Age, 0.8 for Weight and 0.65 for $C M V\left(\mathrm{RRR}_{\text {years }}=\mathrm{RR}_{\text {year }}=2005 / \mathrm{RR}_{\text {year }=1992,} \mathrm{RRR}_{\text {timeon } C M V}=\mathrm{RR}_{\text {time }}=9 \mathrm{~h} / \mathrm{RR}_{\text {time }}=0.3 \mathrm{~h}, \mathrm{RRR}_{\text {age }}=\mathrm{RR}_{\text {age }=31 \text { weeks }} / \mathrm{RR}_{\text {year }}=26\right.$ weeks , $\left.\mathrm{RRR}_{\text {weight }}=\mathrm{RR}_{\text {weight }=1.5 \mathrm{~kg}} / \mathrm{RR}_{\text {year }}=0.7 \mathrm{~kg}, \mathrm{RRR}_{\text {incidenceo } f C L \text { Din } C M V=0.75} / \mathrm{RR}_{\text {incidence }}=0.08\right)$ 
Table 3 Multivariable linear regression analysis

\begin{tabular}{|c|c|c|c|c|c|c|c|}
\hline & \multirow{2}{*}{$\begin{array}{l}\text { Adjusted } \\
\text { B }\end{array}$} & \multirow[b]{2}{*}{ Sig. } & \multicolumn{2}{|c|}{$95 \%$ confidence interval } & \multirow[b]{2}{*}{ RRR } & \multicolumn{2}{|c|}{$95 \%$ confidence interval } \\
\hline & & & $\begin{array}{l}\text { Lower } \\
\text { boundary }\end{array}$ & $\begin{array}{l}\text { Upper } \\
\text { boundary }\end{array}$ & & $\begin{array}{l}\text { Lower } \\
\text { boundary }\end{array}$ & $\begin{array}{l}\text { Upper } \\
\text { boundary }\end{array}$ \\
\hline & Model A & & & & & & \\
\hline \multicolumn{8}{|l|}{$C L D$} \\
\hline (Constant) & -0.66 & 0.900 & -13.03 & 11.70 & & & \\
\hline SensorM & -0.04 & 0.884 & -0.75 & 0.66 & 0.96 & 0.47 & 1.94 \\
\hline TimeCMV & -0.02 & 0.903 & -0.38 & 0.34 & 0.85 & 0.04 & 19.22 \\
\hline Age & 0.03 & 0.850 & -0.36 & 0.42 & 1.17 & 0.16 & 8.32 \\
\hline HLVS & -0.88 & 0.306 & -2.80 & 1.04 & 0.42 & 0.06 & 2.84 \\
\hline LPVS & 0.70 & 0.506 & -1.73 & 3.14 & 2.02 & 0.18 & 23.12 \\
\hline \multicolumn{8}{|l|}{ Death or CLD } \\
\hline (Constant) & -1.86 & 0.412 & -7.22 & 3.49 & & & \\
\hline SensorM & -0.17 & 0.309 & -0.55 & 0.21 & 0.85 & 0.58 & 1.24 \\
\hline TimeCMV & 0.01 & 0.722 & -0.05 & 0.06 & 1.07 & 0.68 & 1.69 \\
\hline Age & 0.08 & 0.299 & -0.09 & 0.25 & 1.47 & 0.62 & 3.47 \\
\hline HLVS & -0.88 & 0.407 & -3.38 & 1.62 & 0.42 & 0.03 & 5.06 \\
\hline \multirow[t]{2}{*}{ LPVS } & 0.68 & 0.127 & -0.28 & 1.65 & 1.98 & 0.76 & 5.19 \\
\hline & Model B & & & & & & \\
\hline \multicolumn{8}{|l|}{$C L D$} \\
\hline (Constant) & 0.07 & 0.904 & -1.21 & 1.35 & & & \\
\hline SensorM & -0.06 & 0.698 & -0.38 & 0.26 & 0.94 & 0.69 & 1.30 \\
\hline HLVS & -0.81 & 0.203 & -2.14 & 0.52 & 0.44 & 0.12 & 1.68 \\
\hline LPVS & 0.72 & 0.011 & 0.21 & 1.23 & 2.06 & 1.23 & 3.43 \\
\hline \multicolumn{8}{|l|}{$\begin{array}{l}\text { Death or CLD } \\
\quad \text { (Constant) }\end{array}$} \\
\hline SensorM & -0.11 & 0.318 & -0.33 & 0.12 & 0.90 & 0.72 & 1.13 \\
\hline HLVS & -0.79 & 0.363 & -2.66 & 1.08 & 0.45 & 0.07 & 2.93 \\
\hline LPVS & 0.46 & 0.089 & -0.09 & 1.01 & 1.59 & 0.92 & 2.74 \\
\hline
\end{tabular}

Multiple linear regression analyses were calculated for chronic lung disease (CLD), defined as on oxygen at 30-36 weeks postgestational age, and death or CLD. The following co-variates were evaluated: SensorM whether or not a Sensormedics type of HFV was used; Time CMV mean time on CMV before start of the study (in hours). HLVS high lung volume strategy in the HFV group, LPVS lung protective ventilation strategy in the CMV group. B was the adjusted estimated coefficient. $R R R$ relative risk ratio= $R_{\text {covariate }=1} / \mathrm{RR}_{\text {covariate }=0}$, for binary variables (SensorM, HLVS and LPVS), for continues variables the extreme values reported in the studies were used, 8.7 for Time

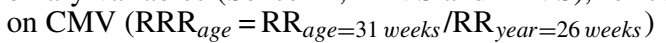

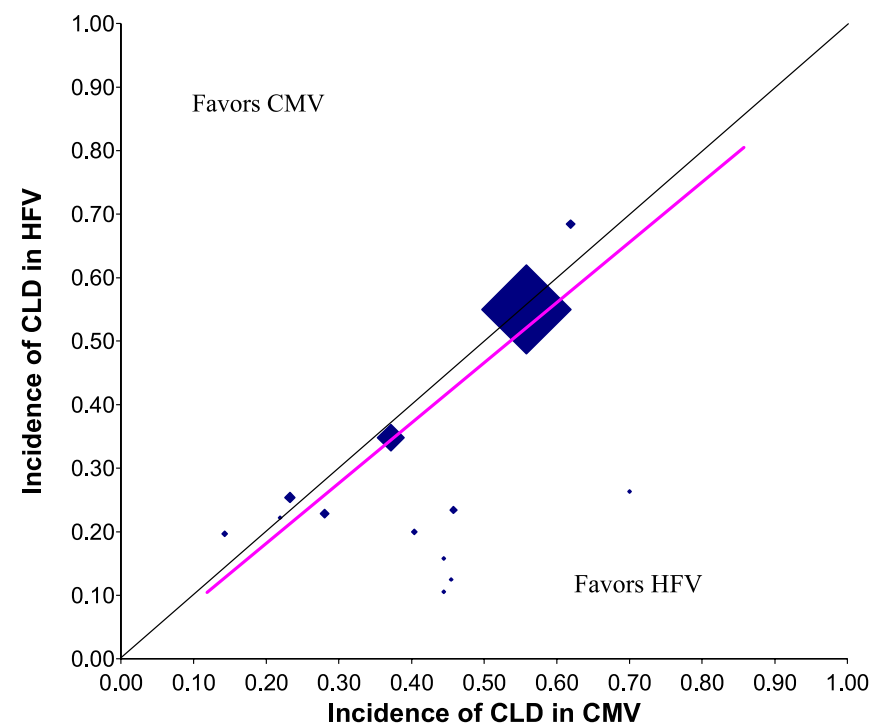

Fig. 5 Linear regression analysis of incidence of CLD in CMV on incidence of CLD in HFV. $y$-axis: incidence of CLD in HFV; $x$-axis: incidence of CLD in CMV. Thin pink line: regression line including all studies was fitted in the model. One study contributed to the fact that surfactant was not used; therefore, surfactant was not used in the multiple linear regression analyses. Two models were fitted. Model A used Sensormedics, time on CMV, gestational age, HLVS, and LPVS as covariates. The largest estimated effects were caused by ventilation strategies, HLVS, and LPVS, adjusted for use of Sensormedics ventilator, prior time on CMV, and gestational age. These estimations were consistent for the outcomes CLD (RRR $=0.42$ and $R R R=2.02$ for HLVS and LPVS, respectively) and death or CLD (RRR $=0.42$ and RRR $=1.98$ fro HLVS and LPVS, respectively). Use of a Sensormedics ventilator seemed to have a much smaller effect on RR for outcome. The RRR of gestational age, comparing 26 weeks with 31 weeks, for CLD and death or CLD were larger $(R R R=1.17$ and $R R R=1.47)$. The effect of a difference in prior time on CMV of $8.7 \mathrm{~h}$ on CLD vs death or CLD was not consistent $(R R R=0.85$ and $\mathrm{RRR}=1.07$, respectively).

A sensitivity analysis was conducted by fitting a second model (model B) with the most important variables, 
HLVS and LPVS, combined with whether or not a Sensormedics ventilator was used. The reported RRRs were comparable to those in the first model. Type of ventilator did not have a large effect compared with ventilation strategies $(R R R=0.94$ and $R R R=0.90)$. The HLVS was associated with a decrease of the RRs comparing HFV with CMV $(R R R=0.44$ and $R R R=0.45)$, while LPVS increased the RRs to the line of no effect $(R R R=2.06$ and $\mathrm{RRR}=1.59$ ).

\section{Discussion}

Our meta-regression analysis showed a clear trend of decreasing differences in pulmonary outcome between $\mathrm{HFV}$ and CMV in randomized trials conducted in premature neonates with IRDS over the years. The most likely hypothesis for this trend was the application of a LPVS in the most recent studies. Use of surfactant could also have a significant contribution, but only one study did not use surfactant [12]. In previous meta-analyses, subgroup analyses or cumulative methods were used to explore heterogeneity $[4,5,7]$. Subgroup analysis is equivalent to meta-regression with a categorical trial-level covariate. Considering subgroup analysis formally as a meta-regression has advantages, since it focuses on differences between subgroups as is appropriate, rather than the effects in each subgroup separately. Furthermore, it is appropriate to use meta-regression to explore sources of heterogeneity, even if an initial overall test for heterogeneity is non-significant. This test often has low power and therefore a non-significant result does not reliably identify lack of heterogeneity [25].

In this meta-regression analysis we evaluated in a quantitative way a number of hypotheses that were raised to account for different results between randomized trials. A relatively large proportion of well-conducted trials were available for the analyses. For most explanatory variables there were important differences among trials. The effects of the two most important covariates, HLVS and LPVS, were consistent in the different models and were even increased in effect size by adjusting for other covariates. None of the competing hypotheses were more likely to influence results as shown by calculating the RRRs. Common pitfalls in meta-regression analysis can occur, such as multiple or post-hoc analyses, and lead to data dredging and a high probability of false-positive conclusions [25]. We, therefore, restricted our analyses to a limited number of pre-specified explanatory covariates.

Publication bias was considered unlikely as an explanation of the apparent diminishing relative effect of HFV. Publication bias is selection bias. If trials are selectively published either because of their size or because of significant results, this would result in an association between trial size and/or precision and the trial outcome. Strictly speaking, funnel plots probe whether studies with little precision (small studies) give different results from studies with greater precision (larger studies). Asymmetry in the funnel plot may therefore result not from a systematic under-reporting of negative trials but from an essential difference between smaller and larger studies that arises from inherent between-study heterogeneity [26]; thus, if larger studies were also associated with changes in ventilation strategies and these strategies resulted in changes in reported RRs, the assumed publication bias would be, in fact, a real association between ventilation strategy and study outcome; therefore, we conditioned the association between precision and effect size, presumably caused by publication bias, on ventilation strategies. This resulted in a lower $p$-value for publication bias and more symmetrical distribution of studies in subgroups in the funnel plots; therefore, what appeared to be publication bias could also be explained by differences in ventilation strategies related to both study size and observed relative risks. However, it should be pointed out that the strength of this evidence is difficult to assess because fewer studies in the subgroups automatically resulted in less power to detect publication bias.

Other alternative hypotheses that have been formulated to explain differences among studies were also less compatible with the evidence [9]. The type of ventilator, Sensormedics vs other types of high-frequency ventilators, displayed RRR close to one. In the crude analyses, prior time on CMV before study initiation showed contradictory effects to what was hypothesized [10]. The adjusted analyses showed conflicting results depending on the outcome. Gestational age and birth weight could also influence the magnitude of the effect of HFV compared with CMV. In the adjusted analysis gestational age did not change the RR for CLD but showed an increase of the RR for less premature neonates. Finally, an increased risk of CLD was not accompanied by a greater relative benefit of HFV as compared with CMV.

The observed effects of continuous variables, such as time on CMV or gestational age, could be exaggerated by small studies with outlying results. For the covariate, time on CMV, the two largest studies showed results that were compatible with the hypothesis that this had no important impact on the results of these trials [2,3]. The same fact applied to the effect of baseline incidence of CLD or death or CLD. Gestational age and weight were comparable between the two largest trials, which made it more difficult to ascertain the relevance of the hypothesis that in smaller and more premature infants HFV performed better than CMV treatment. The observed direction of the effect of gestational age and birth weight, however, was opposite to what the hypothesis predicted. If gestational age was to be interpreted as a higher risk of acquiring CLD, one would expect that an increase in the incidence of CLD was associated with a relatively lower incidence of CLD in HFV treated patients; however, linear regression analysis showed perfectly equal increase in both treatment groups. Still, the 
possibility remains that the relationship with patient averages, such as gestational age and birth weight, across trials was not the same as the relationship for patients within trials, and therefore an effect of these patient characteristics cannot be excluded but only considered in relation to other covariates [25].

Similar findings of the effects of ventilation strategies have been reported by us and other authors as well $[4,5]$; however, meta-analyses are subject to bias when differences among trials are used to explain differences in reported RRs. In this meta-regression analysis we were able to estimate adjusted association measures, thereby diminishing the effects of possible confounders/effect-modifiers. By calculating less biased estimates of the effects of ventilation strategies and the effect of using a Sensormedics ventilator instead of other ventilators on the outcome in the different HFV trials we were able to reinforce the hypothesis that ventilation strategies are more important than type of ventilator to prevent CLD.

The results of this meta-analysis stresses the importance of using appropriate ventilation strategies to prevent ventilator-induced lung damage in a highly vulnerable group of patients; therefore, in clinical practice the question of how to use the ventilator is more important than the question of which ventilator should be used. The major theoretical advantage of HFV to CMV is delivery of smaller tidal volumes to an optimally recruited lung. As this meta-regression analysis did not confirm that subgroups of more premature neonates, avoidance of CMV prior to initiating HFV, or neonates with higher risk of CLD were more likely to benefit form elective HFV in IRDS, future research should be directed at identifying patients in whom HFV does have a benefit over CMV. To improve the robustness of these conclusions and to avoid the limitations of meta-analysis of trials, an individual-patient-data-based meta-regression analysis should be conducted.

\section{Conclusion}

In conclusion, confining randomized trails to smaller or more premature children with IRDS did not seem to result in better pulmonary outcomes of HFV compared with CMV. A generally held opinion that a prolonged ventilation time on CMV prior to initiating HFV diminished the benefits of HFV was not in agreement with the current evidence. The most important effects resulting in differences among trials were probably caused by ventilation strategies applied in HFV- and CMV-treated patients.

\section{References}

1. Froese AB, Kinsella JP (2005) Highfrequency oscillatory ventilation: lessons from the neonatal/pediatric experience. Crit Care Med 33:S115-S121

2. Courtney SE, Durand DJ, Asselin JM, Hudak ML, Aschner JL, Shoemaker CT (2002) High-frequency oscillatory ventilation versus conventional mechanical ventilation for very-low-birth-weight infants. N Engl J Med 347:643-652

3. Johnson AH, Peacock JL, Greenough A, Marlow N, Limb ES, Marston L, Calvert SA, United Kingdom Oscillation Study Group (2002) High-frequency oscillatory ventilation for the prevention of chronic lung disease of prematurity. N Engl J Med 347:633-642

4. Bollen CW, Uiterwaal CS, van Vught AJ (2003) Cumulative metaanalysis of high-frequency versus conventional ventilation in premature neonates. Am J Respir Crit Care Med 168:1150-1155

5. Thome UH, Carlo WA, Pohlandt F (2005) Ventilation strategies and outcome in randomized trials of high frequency ventilation. Arch Dis Child Fetal Neonatal 90(6):466-473
6. Clark RH, Dykes FD, Bachman TE, Ashurst JT (1996) Intraventricular hemorrhage and high-frequency ventilation: a meta-analysis of prospective clinical trials. Pediatrics 98:1058-1061

7. Henderson-Smart DJ, Bhuta T, Cools F, Offringa M (2003) Elective high frequency oscillatory ventilation versus conventional ventilation for acute pulmonary dysfunction in preterm infants. Cochrane Database Syst Rev:CD000104

8. Thome UH, Carlo WA (2000) Highfrequency ventilation in neonates. Am J Perinatol 17:1-9

9. Stark AR (2002) High-frequency oscillatory ventilation to prevent bronchopulmonary dysplasia: Are we there yet? N Engl J Med 347:682-684

10. Rimensberger PC, Beghetti M, Hanquinet S, Berner M (2000) First intention high-frequency oscillation with early lung volume optimization improves pulmonary outcome in very low birth weight infants with respiratory distress syndrome. Pediatrics 105:1202-1208

11. Jadad AR, Moore RA, Carroll D, Jenkinson C, Reynolds DJ, Gavaghan DJ, McQuay HJ (1996) Assessing the quality of reports of randomized clinical trials: Is blinding necessary? Control Clin Trials 17:1-12
12. Clark RH, Gerstmann DR, Null DM Jr, deLemos RA (1992) Prospective randomized comparison of high-frequency oscillatory and conventional ventilation in respiratory distress syndrome. Pediatrics 89:5-12

13. Gerstmann DR, Minton SD, Stoddard RA, Meredith KS, Monaco F, Bertrand JM, Battisti O, Langhendries JP, Francois A, Clark RH (1996) The Provo multicenter early high-frequency oscillatory ventilation trial: improved pulmonary and clinical outcome in respiratory distress syndrome [see comments]. Pediatrics 98:1044-1057

14. Wiswell TE, Graziani LJ, Kornhauser MS, Cullen J, Merton DA, McKee L, Spitzer AR (1996) Highfrequency jet ventilation in the early management of respiratory distress syndrome is associated with a greater risk for adverse outcomes. Pediatrics 98:1035-1043

15. Keszler M, Modanlou HD, Brudno DS, Clark FI, Cohen RS, Ryan RM, Kaneta MK, Davis JM (1997) Multicenter controlled clinical trial of high-frequency jet ventilation in preterm infants with uncomplicated respiratory distress syndrome [see comments]. Pediatrics 100:593-599 
16. Rettwitz-Volk W, Veldman A, Roth B, Vierzig A, Kachel W, Varnholt V, Schlosser R, Loewenich V von (1998) A prospective, randomized, multicenter trial of high-frequency oscillatory ventilation compared with conventional ventilation in preterm infants with respiratory distress syndrome receiving surfactant [see comments]. J Pediatr 132:249-254

17. Plavka R, Kopecky P, Sebron V, Svihovec P, Zlatohlavkova B, Janus V (1999) A prospective randomized comparison of conventional mechanical ventilation and very early high frequency oscillatory ventilation in extremely premature newborns with respiratory distress syndrome. Intensive Care Med 25:68-75

18. Thome U, Kossel H, Lipowsky G, Porz F, Furste HO, GenzelBoroviczeny $\mathrm{O}$, Troger $\mathrm{J}$ Oppermann HC, Hogel J, Pohlandt F (1999) Randomized comparison of high-frequency ventilation with high-rate intermittent positive pressure ventilation in preterm infants with respiratory failure [see comments]. J Pediatr 135:39-46
19. Durand DJ, Asselin JM, Hudak ML, Aschner JL, McArtor RD, Cleary JP, VanMeurs KP, Stewart DL, Shoemaker CT, Wiswell TE, Courtney SE (2001) Early high-frequency oscillatory ventilation versus synchronized intermittent mandatory ventilation in very low birth weight infants: a pilot study of two ventilation protocols. J Perinatol 21:221-229

20. Moriette G, Paris-Llado J, Walti H, Escande B, Magny JF, Cambonie G, Thiriez G, Cantagrel S, LacazeMasmonteil T, Storme L, Blanc T, Liet JM, Andre C, Salanave B, Breart G (2001) Prospective randomized multicenter comparison of high-frequency oscillatory ventilation and conventional ventilation in preterm infants of less than 30 weeks with respiratory distress syndrome. Pediatrics 107:363-372

21. Craft AP, Bhandari V, Finer NN (2003) The sy-fi study: a randomized prospective trial of synchronized intermittent mandatory ventilation versus a highfrequency flow interrupter in infants less than 1000 g. J Perinatol 23:14-19
22. Schreiber MD, Gin-Mestan K, Marks JD, Huo D, Lee G, Srisuparp P (2003) Inhaled nitric oxide in premature infants with the respiratory distress syndrome. N Engl J Med 349:2099-2107

23. Van Reempts P, Borstlap C, Laroche S, Van der Auwera JC (2003) Early use of high frequency ventilation in the premature neonate. Eur J Pediatr 162:219-226

24. Vento G, Matassa PG, Ameglio F, Capoluongo E, Zecca E, Tortorolo L, Martelli M, Romagnoli C (2005) HFOV in premature neonates: effects on pulmonary mechanics and epithelial lining fluid cytokines. A randomized controlled trial. Intensive Care Med 31:463-470

25. Thompson SG, Higgins JP (2002) How should meta-regression analyses be undertaken and interpreted? Stat Med 21:1559-1573

26. Lau J, Ioannidis JP, Terrin N, Schmid CH, Olkin I (2006) The case of the misleading funnel plot. Br Med J 33:597-600 